\title{
Sex Difference in Confidence on Conformity patterns in Female-dominate Stereotyped Task
}

\author{
Zixin $\mathrm{Gu}^{1, *}$, Yuyao Liu ${ }^{2, *}$, Yueyao Yang ${ }^{3, *}$ \\ ${ }^{1}$ College of Liberal Arts and Science, University of Connecticut, Storrs, CT, 06269-3088, United States. \\ Corresponding author. \\ ${ }^{2}$ College of Liberal Arts and Science, University of Connecticut, Storrs, CT, 06269-3088, United States. \\ Corresponding author. \\ ${ }^{3}$ Liberal Arts College, Lawrence University, Appleton, WI, 54911, United States. \\ ${ }^{1}$ Email: zixin.gu@uconn.edu; Email: yuyao.liu@uconn.edu; \\ Corresponding author. ${ }^{3}$ Email: yueyao.yang@lawrence.edu ;
}

\begin{abstract}
Background: The manifestation of gender differences in different aspects has always been a social phenomenon that researchers are interested in and mainly focus on. In Catharine P. Cross's previous research, researchers' study results have proposed there are gender differences in confidence level on conformity patterns as men are significantly more confident in a male-dominated stereotyped cognitive task than women and rely less on social information. However, this study fails to focus on whether gender differences still affect people's self-confidence level in women-dominated stereotyped cognitive tasks. This article will focus on sex differences in confidence influence patterns of conformity, especially in a female-dominated stereotyped cognitive task. We will use an experiment to test whether a stereotyped task that is assumed to favor women (Verbal Memory Task) would induce lower confidence and hence greater reliance on social information in men than women. The experiment found out that men were generally more confident than women in even female-dominated stereotyped cognitive tasks. Therefore, society should provide more education and opportunities to increase women's self-confidence effectively.
\end{abstract}

Keywords: Sex difference, Confidence level, Conformity patterns, Verbal Memory Task

\section{INTRODUCTION}

Our research question is based on Catharine $\mathrm{P}$. Cross's research, which was published in 2016, regarding to sex difference in confidence influence patterns of conformity. This research aims to test the hypothesis that in a task that shows sex difference in confidence, an indirect effect of sex on social information use will be evident [1]. In the experiment, the researchers asked the participants to complete both mental rotation and letter transformation tasks. Mental rotation (MR) tasks have been proved as male domain, while there hasn't been strong evidence to demonstrate that letter transformation tasks have a significant relationship with gender difference. After analyzing data they collected in the experiment, researchers found that in both tasks, participants were more likely to change their answers in response to social information when they expressed low confidence in their initial answer.
Another finding is that women have lower confidence than men in the MR tasks. It may indirectly demonstrate that women feel less confident when they do the tasks which are characterized as "male-dominated". We are inspired by these findings and decided to research whether men will be less confident than women when they do "female-dominated" stereotyped cognitive tasks. Also, we're interested in knowing whether men will perform more reliant on social information when they are doing these tasks. Thus, we decided to compare female and male's confidence performance in stereotyped-tasks on measures of confidence and reliance on social information in a female-dominated stereotyped cognitive task.

\subsection{Topics or Issues}

The effect of gender-related stereotyped tasks on the confidence of males and females in their own initial answers, and the effect of social information provided in 
that specific gender stereotyped task on people's behavior of changing answers because of the lack of confidence. We would cover topics above, and the overall definition of gender-related stereotypes.

\subsection{Sex Difference}

Oswald states that sex differences in one's confidence level of his or her ability or performance can be influenced by gender stereotypes [2]. In certain situation, when gender stereotypes make people consider that one sex is less competent, it will lead to lower level of confidence in that group of people. Cross mentions in her research that women are perceived to have lower confidence than men in various areas [1]. For example, female university students tend to underrate their performance in economics, medicine, and biology [1]. In addition, Picucci's research article published in 2011 found out that women also under-rate their scores when they participate in some psychological experiments like simple navigation tasks on a computer screen [3]. Cross uses "Mental Rotation tasks" which has been found to be male-dominated, and "Letter Transformation tasks" which has been found with no specific gender bias [1]. We decided to do a similar way of testing but using Verbal Memory (VM) tasks, which has been proved by Choi. J's article as a stereotyped female-dominated task [4].

\subsection{Confidence}

"Confidence is a strong predictor of using social information" because lack of confidence in one's own ability can increase the likelihood of relying on social information. Cross also mentions that self-reported confidence level can be influenced by features like ability, performance, and gender stereotypes [1]. This concept shows that people with high ability or performance tend to have higher confidence, but people with low ability or performance tend to have lower confidence. Gender stereotypes factors were mentioned in sex difference part above.

\subsection{Conformity Patterns}

Conformity patterns also known as social information in Cross research. The most common form that social information appears in our life could be social learning, when people's own experiences are insufficient, they tend to seek social information to find a solution effectively [1]. In Cross's research and our own experiment, social information would be the answer from previous participants, so called demonstrators which can give participants reference to decide whether to change their initial answers or not. There is a prediction that the correlation between confidence and the level of relying on social information is negative [1]. This means that low confidence in the initial answer would increase participants' level of relying on social information in both genders.

\section{METHOD}

\subsection{Participant and Task}

Based on the Hausmann M.'research (2009) and Izzaty, R. E. 's research (2019), the Verbal Memory (VM) task has been verified as a gender-stereotyped cognitive task with significant female dominance and continues to show female advantages [5-6]. In Izzaty, R. E. 's research (2019), the research results found out that in the mean verbal ability subtest score comparison, female students have a higher mean score than man students [6]. We will use the methodology of the experiment and use our current resources to connect students in CIS Program and at least recruit 50 of them, including 25 females and 25 males, as our sample. Their age will all be around the same period. All participants we recruit will be right-handers and have no psychological, neurological, and endocrinological disorders. We will remove participants with a psychology-related background and divide all participants into groups of 2-10 people for each experiment. We chose students as our participants because we are interested in researching people of our age, which could represent young people in China.

\subsection{1.he Verbal Memory Task}

Participants are briefed on the experiment as they enter the test room. After participants sit in the back of their computer screen, we read aloud the instructions that appear on their computer screen. Participants will also be told that we will be rewarded based on the accuracy of the final answers. After the verbal fluency task, participants were asked about their gender, whether and the degree they felt pressure in the experiment and describe their strategies to choose whether to use social information. We will eventually obtain informed consent from all participants. The Verbal Memory (VM) task we will use based on the original VM task in Scheuringer, A. 's research article (2017) on sex differences in verbal fluency and VM task in Marco Hirnstein' research article (2015) on gender stereotyping enhances verbal fluency and verbal memory performance in men (and women) [7-8].

\subsubsection{Social Information:}

We will provide participants with a randomly selected number $(\mathrm{N}=2,4$, or 6$)$ of answers from pretest participants. The participants in the main experiment will be informed about this situation. A conditional information lottery will be produced to manipulate the social information. To avoid deception, participants will 
be told that some of the social information is real, and some is not real.

\subsection{Experiment Procedure:}

We consider that it may be very difficult to define the social information that the participants must rely on in the VF task, which impacts their confidence. This has already been discussed in Catharine P. Cross's research (2016). As the participants can answer freely in the VF task, it may be hard to present contradictory social information. To solve this problem, we may change the Verbal Fluency (VF) task to Verbal Memory (VM) task. In Sundermann, E. E. 's research article (2016), the result indicated that the research data shows that women outperform men on verbal memory tasks despite similar levels of brain metabolism and that this advantage may be lifelong [9].

Since verbal memory involves recalling the details of the given information, in our experiment, we will find a medium-length text as shown in Figure 1 for participants to read at a set time (we will do a pretest to set an appropriate time for participants to react and remember the information we give). For example, it could be a paragraph in some news, which contains some details about years, names, and some statistics (we think they are easier to remember). Then, we will delete three or four details or leave them blank and ask the participants to complete them through their memory. It's like fill-in-the-blank games. But we will give them four choices, which have similar meanings and what they need to do is choose one they think is correct to fill in the blanks. When participants completed their tasks for the first time, they were asked, "How confident are you about your answers? and set your level of confidence from 1 (minimum, "completely unconfident") to 4 (highest degree, "very confident"). The next step is that we will give them some social information, such as the answers that other people do in the pre-test. However, we will not tell them whether the answers are wrong or right, and they are only references. After reviewing other people's answers, the participants can choose to change their answers or not. After all the participants finish doing their tasks, we will compare their original answers and final answers to see whether they will change their answers because of the social information we give in the middle of the test. Finally, we will calculate the percentage of women who change their answers and the percentage of men who change their answers and compare these data.

A person who agrees to serve as _ _ 1 _ between two warring factions at the request of both abandons by so agreeing the right to take sides later. To take sides at a later point would be to suggest that the earlier presumptive__ _ was a sham.

\section{Question 1: \\ $\begin{array}{llll}\text { A. Transformer } & \text { B. Media } & \text { C. Mediator } & \text { D. Indicate }\end{array}$}

\section{Question 2: \\ $\begin{array}{lllll}\text { A. Impossible } & \text { B. contradictory } & \text { C. methodology } & \text { D. Impartiality } & \text { E. Training }\end{array}$}

Figure 1, sample question for the experiment

\subsubsection{Experiment Steps:}

First, to test participants' verbal memory abilities, we designed 3 sets of questions and each set consisted of one word question and one text question. These are examples in a question set. But before the formal test began, we gave them a pre-test to get familiar with the whole procedure and the pattern of the question and it's a kind of practice to make sure that they can start answering the question as soon as possible.

Then, we showed them the formal 3 question sets.
For the word memory question, each word appeared $0.8 \mathrm{~s}$ and they had $7 \mathrm{~s}$ to answer the question; for the text memory question, each medium-length sentence appeared $15 \mathrm{~s}$ and they had $15 \mathrm{~s}$ to answer the question. They needed to write them down on the paper we gave. After they fill in their answers in the paper, they need to report how confident they are in the answers. We designed 7 levels, from very unconfident to very confident, and they chose one from these 7 levels according to their own real feelings when they did the questions. So, this is basically the first step. In addition, before the participants started doing the experiment, 
they were asked to give simple information about their backgrounds. For example, their gender, age, handedness, education level, and whether they have memory disorders.

In this step, the independent variable here is sex difference and the dependent variable is confidence level. Which means the difference on sex will trigger the change of confidence level. We will discuss more when we explain our results.

Next is our second step. After the participants completed the verbal memory tasks and evaluated their confidence level. Then, we gave them the social information, which is the answers that other students gave in the pre-tests. But they were randomly assigned. For example, some participants got 2 social information, some got 4 and some got 6 . Then we gave back the paper with their original answers. They could choose to change their answers according to the social information, or other people's answers or keep their original answers. We gave them half of a minute to fix answers. Then, they were asked to evaluate their confidence level again like what we did on the first step.

What we compare here is the change of their confidence level after seeing other people's answers. In other words, it will rely on social information and trigger the change of the confidence level.

\section{DATA ANALYSIS AND RESULTS}

Since many people in the CIS program are interested in our experiment and willing to participate, we collect 50 participants (25 males \& 25 females). First, we deleted the one who did not complete the experiment. Then, some of the participants' ages are too young that they may not understand the whole procedure, while some of the participants' ages are much older than we expected. They may speculate about our experiment and cover their true thoughts to us. So, we delete those 9 samples. We also delete the 7 samples from participants who have never had an English education experience since our VM tasks are all English and they may not understand the experiment. Finally, to test whether the participants take our experiment seriously, we set the first set of the word questions incredibly easy. We got 12 males and 12 females.

All data were analyzed with Excel using a one-way analysis of variance. This approach is used mainly to test whether there is a significant difference in the mean of a dependent variable of multiple samples affected by a single factor. We chose the one-way analysis of variance because even though there are multiple dependent variables in our experiment (e.g., confidence level, rely on social information), there is only one independent variable, sex difference. We also generate 95\% credible intervals (CI) for each parameter. Strong evidence of a relationship between variables was inferred when CIs for a parameter value did not include zero.

$\begin{aligned} & \text { Confidence Change Level in Gender } \\
& \text { Anova: Single Factor } \\
& \text { SUMMARY }\end{aligned}$
\begin{tabular}{lrrrrr}
\multicolumn{1}{c}{ Groups } & Count & Sum & Average & Variance \\
\hline Male & 12 & 3 & 0.25 & 0.38636364 \\
Female & 12 & 13 & 1.08333333 & 1.17424242 \\
\hline
\end{tabular}

\begin{tabular}{|c|c|c|c|c|c|c|}
\hline Source of Variation & SS & $d f$ & $\mathrm{MS}$ & $\bar{F}$ & $P$-value & F crit \\
\hline Between Groups & 4.16666667 & & 14.16666667 & 5.33980583 & 0.03060109 & 4.3009495 \\
\hline Within Groups & 17.1666667 & & 220.78030303 & & & \\
\hline Total & 21.3333333 & & 23 & & & \\
\hline
\end{tabular}

Figure 2, The significance of gender difference in confidence change level 


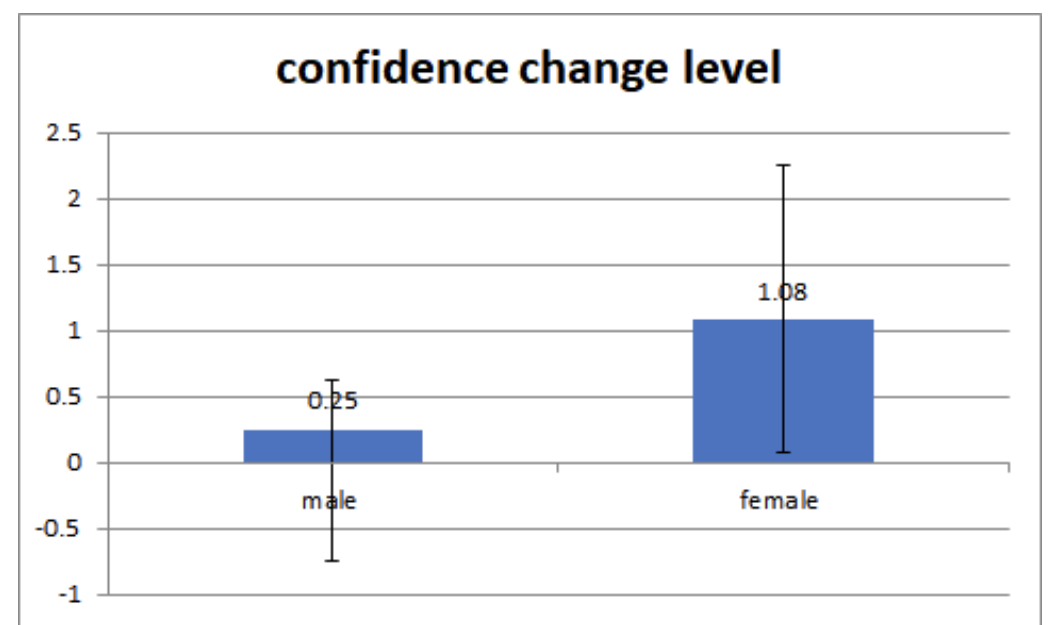

(Figure 3, Histogram of gender difference in confidence change level; $\mathrm{P}<0.05$ )

In the first set of analyses, all the confidence level trials from the VM $(\mathrm{N}=24)$ task were analyzed. We take participants' sex as the independent variable and the confidence change level as the dependent variable and then estimate the effects of participant sex on confidence. There is an obvious significance in the Confidence Change Level in Male Anova: Single Factor

\begin{tabular}{lrrcc}
\multicolumn{1}{l}{ SUMMARY } \\
\hline \multicolumn{1}{c}{ Groups } & Count & Sum & Average & Variance \\
\hline Before & 12 & 46 & 3.83333333 & 1.06060606 \\
After & 12 & 49 & 4.08333333 & 1.17424242 \\
\hline
\end{tabular}

\begin{tabular}{|c|c|c|c|c|c|c|}
\hline Source of Variation & SS & df & MS & $\mathrm{F}$ & P-value & F crit \\
\hline Between Groups & 0.375 & 1 & 0.375 & 0.33559322 & 0.56826909 & 4.3009495 \\
\hline Within Groups & 24.5833333 & 22 & 1.11742424 & & & \\
\hline Total & 24.9583333 & 23 & & & & \\
\hline
\end{tabular}

results (Figure 2). It reported in Figure 3 that men gave a higher level of confidence in their answers than women $(\mathrm{P}$-value $=0.0306)$.

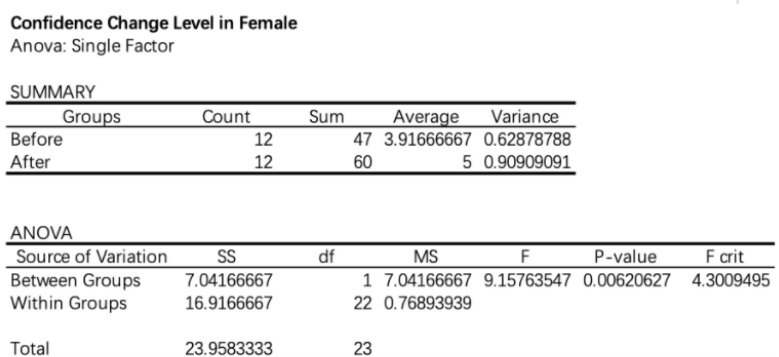

(Figure 4, Change in self-confidence within each gender group)

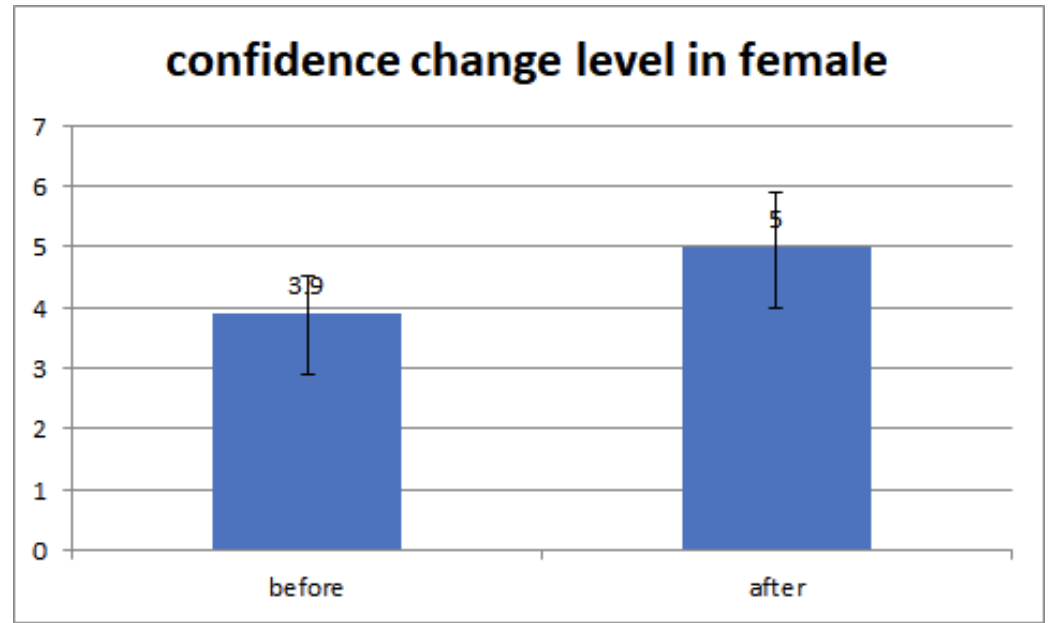

(Figure 5, degree of confidence changes within female group)

To get a better understanding about these results we set the second analyses (Figure 4). We analyzed the confidence change level before and after the VM task within the male group and the confidence change level before and after the VM task within the female group. There is no obvious significance in the male's confidence change level. Contrary to the male's confidence level, there is an obvious significance between the female's confidence change level. These results support our previous results, it also shows that the difference in confidence change level between the male and female is mainly due to the female's confidence change level (Figure 5). 
Level of Relying on Social Information in Gender

Anova: Single Factor

SUMMARY

\begin{tabular}{lrrcc}
\hline \multicolumn{1}{c}{ Groups } & \multicolumn{1}{c}{ Count } & Sum & Average & Variance \\
\hline Male & 12 & 14 & 1.16666667 & 1.24242424 \\
Female & 12 & 17 & 1.41666667 & 1.90151515 \\
\hline
\end{tabular}

\begin{tabular}{|c|c|c|c|c|c|c|}
\hline Source of Variation & SS & $\mathrm{df}$ & $\mathrm{MS}$ & $\bar{F}$ & $P$-value & F crit \\
\hline Between Groups & 0.375 & 1 & 0.375 & 0.23855422 & 0.63008531 & 4.3009495 \\
\hline Within Groups & 34.5833333 & 22 & 1.5719697 & & & \\
\hline Total & 34.9583333 & 23 & & & & \\
\hline
\end{tabular}

(Figure 6, gender difference in degree of reliance on social information)

In the third set of analyses, all the degrees of reliance on social information trials from the VM $(\mathrm{N}=$ 24) task were analyzed. We take participants' sex as the independent variable and degrees of reliance on social information as the dependent variable and then estimate the effects of participants sex on degrees of reliance on social information. We found that there is no obvious significance in this result (Figure 6). We found that the main reason for this outcome is that the degree of reliance on social information is the ratings made by the participants themselves. So, it is very likely that their self-report may not be true.

\section{CONCLUSION}

In the VM task, men had higher average confidence than women, supporting the previous study. Notably, the sex difference in confidence in the VM task existed independently from the relationship between sex and accuracy. Thus, these analyses still provide clear evidence supporting that sex differences may affect an individual's confidence level in a gender-stereotyped task. Moreover, even though the evidence for a sex difference in the degree of reliance on social information was weak, the previous study supports evidence for a sex difference in confidence, an indirect effect of sex on social influence.

\section{DISCUSSION}

After this experiment, we found out that women usually underestimate their abilities even in their dominant area. They are more susceptible to the thoughts of others in their environment. We suggest that schools should provide more educational programs to enhance their confidence in academics. The various colleges and universities should set up many education programs to cultivate women's independence and improve women's self-esteem and self-improvement. We also suggest that society should provide more opportunities for females, especially in the workplace. Providing women with more positions and job opportunities to demonstrate their abilities and to increase women's confidence in the workplace.

For the future experiment, we need to enlarge our sample size to realize the current experimental results further. Also, we need to provide a better experimental environment and reduce the influence of environmental factors on the experimental results. For this experiment, we focus more on participants' attitudes toward social information when it supports their answers. In the future study, we need to focus more on the social information that may contradict participants' answers.

\section{REFERENCES}

[1] Cross, C. P., Brown, G. R., Morgan, T. J. H., \& Laland, K. N. (2017). Sex differences in confidence influence patterns of conformity. The British Journal of Psychology, 108(4), 655-667. https://doi.org/10.1111/bjop.12232

[2] Oswald, D. L. (2008). Gender stereotypes and women's reports of liking and ability in traditionally masculine and feminine occupations. Psychology of Women Quarterly, 32, 196-203. doi:10.1111/j.1471-6402.2008.00424.x

[3] Picucci, L., Caffo, A. O., \& Bosco, A. (2011). Besides navigation accuracy: Gender differences in strategy selection and level of spatial confidence. Journal of Environmental Psychology, 31, 430 438. doi:10.1016/j.jenvp.2011.01.005

[4] Choi, J., \& L'Hirondelle, N. (2005). Object location memory: A direct test of the verbal memory hypothesis. Learning and Individual Differences, 15(3), 237-245. https://doi.org/10.1016/j.lindif.2005.02.001

[5] Hausmann, M., Schoofs, D., Rosenthal, H. E., \& 
Jordan, K. (2008). Interactive effects of sex hormones and gender stereotypes on cognitive sex differences-A psychobiosocial approach. Psychoneuroendocrinology, 34(3), 389-401. https://doi.org/10.1016/j.psyneuen.2008.09.019

[6] Izzaty, R. E., \& Setiawati, F. A. (2019, August). Influence of Educational Level and Gender on Students' Verbal Ability. In 1st International Conference on Education Social Sciences and Humanities (ICESSHum 2019) (pp. 156-165). Atlantis Press.

[7] Scheuringer, A., Wittig, R., \& Pletzer, B. (2017). Sex differences in verbal fluency: the role of strategies and instructions. Cognitive Processing, 18(4), 407-417. https://doi.org/10.1007/s10339017-0801-1

[8] Hirnstein, M., Freund, N., \& Hausmann, M. (2012). Gender Stereotyping Enhances Verbal Fluency Performance in Men (and Women). Zeitschrift Für Psychologie, 220(2), 70-77. https://doi.org/10.1027/2151-2604/a000098

[9] Sundermann, E. E., Maki, P. M., Rubin, L. H., Lipton, R. B., Landau, S., \& Biegon, A. (2016). Female advantage in verbal memory: Evidence of sex-specific cognitive reserve. Neurology, 87(18), 1916-1924.

https://doi.org/10.1212/WNL.0000000000003288 